\title{
A reasoning approach to introspection and unawareness
}

Citation for published version (APA):

Gossner, O., \& Tsakas, E. (2010). A reasoning approach to introspection and unawareness. METEOR, Maastricht University School of Business and Economics. METEOR Research Memorandum No. 006 https://doi.org/10.26481/umamet.2010006

Document status and date:

Published: 01/01/2010

DOI:

10.26481/umamet.2010006

Document Version:

Publisher's PDF, also known as Version of record

\section{Please check the document version of this publication:}

- A submitted manuscript is the version of the article upon submission and before peer-review. There can be important differences between the submitted version and the official published version of record.

People interested in the research are advised to contact the author for the final version of the publication, or visit the DOI to the publisher's website.

- The final author version and the galley proof are versions of the publication after peer review.

- The final published version features the final layout of the paper including the volume, issue and page numbers.

Link to publication

\footnotetext{
General rights rights.

- You may freely distribute the URL identifying the publication in the public portal. please follow below link for the End User Agreement:

www.umlib.nl/taverne-license

Take down policy

If you believe that this document breaches copyright please contact us at:

repository@maastrichtuniversity.nl

providing details and we will investigate your claim.
}

Copyright and moral rights for the publications made accessible in the public portal are retained by the authors and/or other copyright owners and it is a condition of accessing publications that users recognise and abide by the legal requirements associated with these

- Users may download and print one copy of any publication from the public portal for the purpose of private study or research.

- You may not further distribute the material or use it for any profit-making activity or commercial gain

If the publication is distributed under the terms of Article $25 \mathrm{fa}$ of the Dutch Copyright Act, indicated by the "Taverne" license above, 


\section{Maastricht University}

Olivier Gossner, Elias Tsakas

A reasoning approach to introspection and unawareness

$\mathrm{RM} / 10 / 006$

\section{METEOR}

Maastricht University School of Business and Economics

Maastricht Research School of Economics

of Technology and Organization

P.O. Box 616

NL - 6200 MD Maastricht

The Netherlands 


\title{
A reasoning approach to introspection and
}

\author{
unawareness*
}

\author{
Olivier Gossner ${ }^{\dagger}$ and Elias Tsakas $\ddagger$
}

February 24, 2010

\begin{abstract}
We introduce and study a unified reasoning process which allows to represent the beliefs of both a fully rational agent and of an unaware one. This reasoning process provides natural properties to introspection and unawareness. The corresponding model for the rational or boundedly rational agents is both easy to describe and to work with, and the agent's full system of beliefs has natural descriptions using a reduced number of parameters.
\end{abstract}

KEYWORDS: Belief, information, reasoning, introspection, unawareness.

JEL Classification: D80, D83, D89.

${ }^{*}$ We are indebted to Alpaslan Akay, Robert Aumann, Geir Asheim, Pierpaolo Battigalli, Giacomo Bonanno, Martin Dufwenberg, Amanda Friedenberg, Joe Halpern, Aviad Heifetz, Barton Lipman, Philippe Mongin, Andrés Perea, Dov Samet, Burkhard Schipper, Jeff Steif, Bernard Walliser, Mark Voorneveld and Jörgen Weibull for fruitful discussions and useful comments. We would also like to thank the audiences of the Game Theory Festival (Stony Book), ESEM (Barcelona), SAET (Ischia), Paris Game Theory Seminar, IHPST (Paris), Bocconi University, University of Warwick, HEC Lausanne and Göteborg University. The financial support from the Adlerbertska Forskningsstiftelsen Foundation and the Marie Curie Intra-European Fellowship are gratefully acknowledged.

${ }^{\dagger}$ Paris School of Economics, and London School of Economics and Political Science; ogossner@ens .fr

${ }_{\ddagger}^{\ddagger}$ Department of Economics, Maastricht University; e.tsakas@maastrichtuniversity.nl 


\section{Introduction}

Modeling the beliefs of an economic agent, whether fully rational or boundedly rational is, many decades after the seminal work of Simon (1955), still a fundamental question open to debate. Typical beliefs of a boundedly rational agent can exhibit delusion or unawareness, while the beliefs of a rational agent are exempt of such phenomena.

We can distinguish two roots to the agent's beliefs: direct observation, which consists of the agent's experiences in different states of the world, and reasoning, which is the process through which the agent completes his system of beliefs using logical deductions. It is apparent from everyday experience that the same agent can be either aware or unaware of the same facts depending on circumstances, and that in most situations, the same agent is both aware of some facts and unaware of others. Hence, awareness and unawareness are not the outcome of distinct reasoning abilities, but rather of distinct experiences. Since the same cognitive capacities are compatible with both awareness and unawareness, a unified reasoning process for both the aware and unaware agents has to be the backbone of a model that accounts for these phenomena.

All models previously introduced in the literature by Modica and Rustichini (1994, 1999), Halpern (2001), Halpern and Rêgo (2008), Heifetz et al. (2006, 2008) and Li (2009) rely on distinct reasoning processes depending on the agent's awareness, or unawareness. We find this problematic since, in order to describe an agent's state of mind in these models, one first needs to start by specifying which propositions the agent is aware or unaware of, then define the agent's reasoning process accordingly, and finally complete the system of the agent's beliefs with the direct observation of phenomena by the agent. This is as if awareness preceded reasoning and direct experience, whereas it should arise as the product of the combination of these.

The aim of this paper is to introduce a model which encompasses both the rational and boundedly rational agent, with the following important features: The reasoning process is the same for the aware and unaware agent. This unified reasoning process not only is compatible with the agent's awareness or unawareness, but also brings them natural properties. The model is both simple and tractable, in the sense that it is easy to describe and easy to work with. Finally, it has low complexity, meaning that the agent's beliefs can be fully described using a limited number of parameters.

In our approach, the agent's beliefs can be of two types: universal, or contingent. Universal beliefs are state independent, such as belief in the rules of logic. Contingent beliefs arise from direct observation, and are state dependent. We say that the agent has 
faith in a proposition if he universally believes this proposition. A key assumption in our model is that the agent has faith in his introspection capacity. This means that the agent has faith in the fact that when he believes a proposition, he also believes that he believes in it (positive introspection), while if he disbelieves a proposition, he also believes that he disbelieves it (negative introspection). Introspection is a strong assumption, one which, for instance, is incompatible with unawareness. Our assumption, however, is distinct, and in fact much weaker than introspection, as it only states that the agent has faith in introspection, while introspection itself may or may not hold.

Studying the system of propositions the agent has faith in, we show that faith in introspection for all propositions is equivalent to faith in introspection for primitive ${ }^{1}$ propositions only. This result allows us to interpret faith in introspection as the assumption that the agent believes in his own familiarity with the relevant primitive phenomena describing his environment.

This first result has interesting implications: Consider the state space in which the agent has faith in introspection on primitives and in being non-delusional ${ }^{2}$ about these primitive propositions, and also is actually capable of introspection for primitive propositions. We show that this state space coincides with $\Omega_{5}$, the benchmark state space for the rational agent (see, for instance, Aumann (1999) or Chellas (1980)) in which the agent is capable of introspection and is non-delusional on every proposition, including all epistemic propositions. Thus, introspection extends from the relatively small set of primitive propositions to the whole set of propositions. This shows that introspection does not need to be a mental process per se: It is the natural consequence of 1) the agent's familiarity with the environment 2) the agent assuming his own familiarity with the environment and 3) the agent's deductive process. This result thus brings a foundation for introspection, which is a central assumption in the literature (Aumann, 1999).

We now turn to the more general case in which the agent has faith in introspection, while introspection may or may not hold. Recall the two main notions of unawareness from the literature: According to Modica and Rustichini (1994, 1999), the agent is unaware of a proposition if negative introspection fails for this proposition, i.e., if the agent does not believe in the proposition, and also does not believe that he disbelieves it. A stronger unawareness concept was introduced by Dekel et al. (1998): The agent is unaware of

\footnotetext{
${ }^{1}$ Primitive propositions are those that do not involve the agent's belief. Notice that our terminology is slightly non-standard, as the set of primitives that we consider is closed under the negation, conjunction and disjunction, e.g., the proposition "Ann has blue eyes or it is raining in New York" is a primitive.

${ }^{2}$ Meaning that whenever the agent believes a primitive proposition is true, this proposition is indeed true.
} 
a proposition if he does not believe in any sequence of "I believe in" and "I disbelieve in" followed by the proposition. This stronger concept formalizes a complete lack of recognition of the proposition, as for instance the agent who is unaware of a proposition cannot believe that he disbelieves that he believes in this proposition. We show that whenever the agent has faith in introspection, the two notions are necessarily equivalent, namely, a failure of negative introspection on some proposition is necessarily accompanied by, and can only be explained by, a total failure of recognition of this proposition.

We study the state space $\Omega_{u}$, superset of $\Omega_{5}$, in which the agent has faith in introspection, while positive introspection (a much better accepted assumption than negative introspection) holds. We show (in Proposition 2) that for states in $\Omega_{u}$ only two cases may arise: Either introspection holds for every proposition, or there exists a primitive proposition that the agent is unaware of. Thus, our state space encompasses both the standard rational agent, for whom introspection holds on every proposition, and the boundedly rational one, who exhibits unawareness on some primitive propositions. The model is also tight as no other possibilities may arise.

It is important, for practical and tracatability reasons, to know how complex the description of the agent's beliefs is in the state space $\Omega_{5}$. Halpern (1995) showed that elements of $\Omega_{5}$ have a simple description, as every state in $\Omega_{5}$ is entirely described by the values of primitive propositions and the agent's beliefs on primitive propositions at that state. Meanwhile, $\Omega_{u}$ being larger than $\Omega_{5}$, and allowing for unawareness, one expects the description of elements of $\Omega_{u}$ to be more complex than in $\Omega_{5}$. This is indeed the case, but elements of $\Omega_{u}$ also have simple description: In $\Omega_{u}$, a state is described by the values of primitive propositions and the beliefs of the agent on any proposition that contains at most once the belief operator. This implies in particular that $\Omega_{u}$ has bounded cardinality if the set of propositions is constructed starting with a finite set of primitive propositions. An alternative, and rather natural, description of elements of $\Omega_{u}$ relies on the set of propositions the agent is aware of: A state in $\Omega_{u}$ is given by the values of the primitive propositions, the agent's beliefs on these primitives, and by the set of propositions the agent is aware of at this state.

Our primary model is a syntactic one, in the tradition of Chellas (1980) and Aumann (1999). Syntactic models explicitly represent the agent's reasoning and belief construction processes. Semantic models, (Hintikka, 1962; Aumann, 1976; Geanakoplos, 1989; Dekel et al., 1998) represent the outcome of this process in the form of a possibility correspondence that assigns to each state of the world the set of states that the agent considers as possible. 
In Section 6, we construct a semantic model which is equivalent to our syntactic model. An alternative way to look at the syntactic model is its semantic form, which retains all the same properties concerning in particular unawareness and the role of primitive propositions.

The paper is organized as follows: Section 2 recalls the model of syntactic beliefs. Section 3 motivates the main questions through an example. We introduce our approach of universal beliefs versus contingent beliefs in Section 4, and study the properties of unawareness in our model in Section 5. We present the model of semantic beliefs in Section 6, and conclude with a discussion in Section 7.

\section{Propositions}

We recall the syntactic model of belief from Aumann (1999), Chellas (1980), and Fagin et al. (1995). We start with a set of primitive propositions, $\Phi_{0}$, which describe facts about the world that do not involve the agent's belief. Examples of primitive propositions are "it is raining" or "Ann has blue eyes".

The symbols $\neg, \vee$ and $\wedge$ express negation, disjunction and conjunction, i.e., $\neg \phi$ stands for "not $\phi$ ",$\left(\phi_{1} \vee \phi_{2}\right)$ stands for " $\phi_{1}$ or $\phi_{2}$ " and $\left(\phi_{1} \wedge \phi_{2}\right)$ stands for " $\phi_{1}$ and $\phi_{2}$ ". The set of primitive propositions $\Phi_{0}$ is closed under these operations: $\phi_{1} \vee \phi_{2}, \phi_{1} \wedge \phi_{2}$ and $\neg \phi_{1}$ are primitive propositions whenever $\phi_{1}, \phi_{2}$ are.

The symbol $\beta$ expresses belief: $\beta \phi$ stands for "the agent believes $\phi$ ".

The set of propositions $\Phi$ is the closure of $\Phi_{0}$ under $\beta, \vee, \wedge$ and $\neg$. It is the smallest set of propositions that can be constructed from $\Phi_{0}$ using these operations. For instance, $\beta\left(\phi_{1} \vee \phi_{2}\right) \wedge \beta \neg \phi_{3}$ is a proposition whenever $\phi_{1}, \phi_{2}, \phi_{3}$ are. Non-primitive propositions, such as $\phi_{1} \vee \beta \phi_{2}$, are called epistemic.

The proposition " $\phi_{1}$ implies $\phi_{2}$ " is denoted by $\left(\phi_{1} \rightarrow \phi_{2}\right)$ and is an abbreviation for $\left(\neg \phi_{1} \vee \phi_{2}\right)$; " $\phi_{1}$ if and only if $\phi_{2}$ " is denoted by $\left(\phi_{1} \leftrightarrow \phi_{2}\right)$ and is defined as $\left(\phi_{1} \rightarrow\right.$ $\left.\phi_{2}\right) \wedge\left(\phi_{2} \rightarrow \phi_{1}\right)$.

For a proposition $\phi$, we say that "the agent satisfies positive introspection for $\phi$ " to denote the proposition $(\beta \phi \rightarrow \beta \beta \phi)$, and "the agent satisfies negative introspection for $\phi$ " denotes the proposition $(\neg \beta \phi \rightarrow \beta \neg \beta \phi)$. As usual, we interpret positive introspection as the agent's belief of his own beliefs, and negative introspection as the agent's belief of what he disbelieves. Finally, the expression "the agent satisfies the truth axiom for $\phi$ " stands for $(\beta \phi \rightarrow \phi)$. 


\section{Motivating example}

We recall the example from Conan Doyle's short story "Silver Blaze". A dialog takes place between the famous detective Sherlock Holmes and the Scottland Yard detective Gregory:

Gregory: Is there any other point to which you would wish to draw my attention?

Holmes: To the curious incident of the dog in the night-time.

Gregory: The dog did nothing in the night-time.

Holmes: That was the curious incident.

From noticing that the dog did not bark in the night-time, Holmes infers that no one intruded in the house. On the other hand, Gregory, who was able to make the same observations as Holmes did, failed to reach this conclusion. This example has received considerable attention in the epistemology literature (see for instance Geanakoplos (1989), Dekel et al. (1998)), and is in particular used to illustrate the implications of unawareness. Our first exercise, which, surprisingly enough, has not been filled in the literature, is to detail the mental process through which Holmes reaches his conclusion. We then discuss the several steps where Gregory may fail to follow Holmes' mental process, as well as modeling questions arising from this example.

\subsection{Holmes: The rational benchmark}

We decompose Holmes' reasoning process in two parts. The first part allows to reach the conclusion that the dog did not bark, while the second part comes to the conclusion that no intruder entered the house.

For the first part, let $B$ denote the proposition "the dog barked", and $\beta_{H}$ Holmes' belief operator. The first premise is that Holmes did not observe the $\operatorname{dog}$ barking: $\neg \beta_{H} B$. The second is that negative introspection applies $\beta_{H} \neg \beta_{H} B$. Next, one needs to assume that Holmes believes that, had the dog barked, he would have heard it: $\beta_{H}\left(B \rightarrow \beta_{H} B\right)$. Using propositional calculus (in particular, the contraposition), Holmes believes that, if he did not believe that the dog had barked then the dog indeed did not bark: $\beta_{H}\left(\neg \beta_{H} B \rightarrow \neg B\right)$. From this, Holmes infers that the dog did not bark: $\beta_{H} \neg B$.

As for the second part, let $I$ denote the proposition "there was an intruder in the house". Holmes assumes that, had an intruder come into the house, the dog would have 
barked: $\beta_{H}(I \rightarrow B)$. Using propositional calculus, Holmes believes that if there was no barking, there was no intruder: $\beta_{H}(\neg B \rightarrow \neg I)$. Finally, Holmes combines his belief in this implication with his belief in the absence of barking to reach the conclusion that no intruder came in the house: $\beta_{H} \neg I$.

\subsection{Gregory: The boundedly rational agent}

Gregory's reasoning may fail to follow that of Holmes in several points. Let $\beta_{G}$ denote Gregory's belief operator. An immediate possibility is that Gregory fails to use the rule of inference adequately: We could have in this case $\beta_{G}(\neg B \rightarrow \neg I), \beta_{G}(\neg B)$, but at the same time $\neg \beta_{G}(\neg I)$. A second possibility is that Gregory is not capable of following the rules of propositional calculus, as for instance is the case if $\beta_{G}(I \rightarrow B)$ and $\neg \beta_{G}(\neg B \rightarrow \neg I)$. A third possibility is that Gregory does not have the same understanding of the world as Holmes, and does not believe that the presence of an intruder would have the dog bark: $\neg \beta_{G}(I \rightarrow B)$.

Finally, the most interesting possibility, and most likely, the one which is implicit in the story, is that Gregory is not aware of the possibility of the $\operatorname{dog}$ barking: $\neg \beta_{G} B$, while $\neg \beta_{G} \neg \beta_{G} B$.

\subsection{Modeling questions}

We aim at developing a model which can encompass both the rational agent (Holmes) and the boundedly rational one (Gregory), while retaining tractability. Let us admit that in the example, the fundamental distinction between Holmes and Gregory is their ability to apply negative introspection to the proposition "the dog barked". This means that we have in mind a model in which negative introspection does not necessarily hold for all propositions, or for all agents. This opens several modeling questions.

First, should introspection be interpreted as a mental process per se? If the answer is yes, it may be difficult to justify that the same agent (e.g., Gregory) is capable of applying this process to some propositions, and incapable of applying it to some others. A good model of beliefs should, as much as possible, explain why introspection can hold for some propositions and not for others, or at least provide a clear interpretation for this fact.

Second, in the case of Holmes, it looks natural that he is aware of a proposition such as "I believe that the dog did not bark", given that he is aware of the dog barking or not. Note that, however, awareness of such a proposition is not used in the reasoning 
process we described for Holmes, and can a priori fail in a model where introspection does not necessarily hold for every proposition. A desirable property of a belief model is that awareness (or introspection capacities) between propositions should be related. This type of relations should be the consequences of natural assumptions of the model, and should not be part of the assumptions themselves.

As we see, having a model in which introspection can hold for some propositions without holding for others - and in which the structure of the set of propositions for which introspection holds has a clear and intuitive structure - is not a straightforward task. We present our approach in the next section.

\section{Our approach: Universal beliefs and contingent be- liefs}

We are ultimately interested in devising a state space, where each state describes the truth value of every proposition, including the agent's beliefs. In our approach, the agent's beliefs can be of two types: Universal or contingent. Universal beliefs consist of beliefs in propositions that are not dependent on any particular state. For instance, belief in a proposition such as "a cat is mortal, or it is not" is universal, as belief in such a proposition is derived from rules of logic, and entails no beliefs about the nature of a cat, or the meaning of being mortal. On the other hand, belief in a proposition such as "it is raining in New York", is contingent by nature. Belief in such a proposition arises from particular observation, at particular states of nature, about the weather in New York, and can differ from one state of nature to the other.

We describe the agent's universal beliefs in Section 4.1, and the state space is presented in Section 4.2.

\subsection{Faith: Universal beliefs}

We describe the set $F$ of propositions that the agent has faith in. When considering a state space model, these propositions will be assumed to be believed by the agent at all states (see Section 4.2).

The set $F$ is constructed from a basic set of propositions using inference rules. The basic set of propositions, else called assumptions, is the set $A$ consisting of the following propositions: 
$\left(A_{0}\right)$ All tautologies of propositional calculus

$\left(A_{I}\right) \beta\left(\phi_{1} \rightarrow \phi_{2}\right) \rightarrow\left(\beta \phi_{1} \rightarrow \beta \phi_{2}\right)$, for every $\phi_{1}, \phi_{2} \in \Phi$ (Axiom of distribution)

$\left(A_{1}\right) \beta\left(\phi_{1} \wedge \phi_{2}\right) \leftrightarrow\left(\beta \phi_{1} \wedge \beta \phi_{2}\right)$, for every $\phi_{1}, \phi_{2} \in \Phi$ (Conjunction of belief)

$\left(A_{2}\right)\left(\beta \phi_{1} \vee \beta \phi_{2}\right) \rightarrow \beta\left(\phi_{1} \vee \phi_{2}\right)$, for every $\phi_{1}, \phi_{2} \in \Phi$ (Disjunction of belief)

$\left(A_{3}\right) \beta \phi \rightarrow \phi$, for every $\phi \in \Phi_{0}$ (Truth axiom on primitive propositions)

$\left(A_{4}\right) \beta \phi \rightarrow \beta \beta \phi$, for every $\phi \in \Phi_{0}$ (Positive introspection on primitive propositions)

$\left(A_{5}\right) \neg \beta \phi \rightarrow \beta \neg \beta \phi$, for every $\phi \in \Phi_{0}$ (Negative introspection on primitive propositions)

$\left(A_{6}\right) \beta \phi \rightarrow \neg \beta \neg \phi$, for every $\phi \in \Phi$ (Consistency of belief)

The first axiom $\left(A_{0}\right)$ refers to obvious propositions, such as $\left(\left(\phi_{1} \rightarrow \phi_{2}\right) \leftrightarrow\left(\neg \phi_{2} \rightarrow \neg \phi_{1}\right)\right)$, which are logically true. The axiom of distribution says that if it is believed that $\phi_{1}$ implies $\phi_{2}$ and it is also believed that $\phi_{1}$ is true, then it is necessarily believed that the logical consequence $\phi_{2}$ is also true. Conjunction says that " $\phi_{1}$ and $\phi_{2}$ " is believed if and only if both propositions are believed. Disjunction states that " $\phi_{1}$ or $\phi_{2}$ " is believed if at least one of the propositions is believed. Consistency says that the agent cannot believe a proposition and its negation simultaneously.

The truth axiom says that the agent is confident that his own beliefs are right, i.e., if he believes a proposition then this proposition is true. Positive and negative introspection are also assumed to be part of the agent's faith for primitive propositions. This means that the agent assumes sufficient familiarity with his own environment: He is confident that he is capable of forming correct beliefs about his own beliefs about primitive propositions, which describe the relevant parameters of his environment.

The inference rules through which propositions in $F$ are constructed from other propositions in $F$, are the following:

$\left(R_{I}\right)$ If $\phi_{1} \in F$ and $\left(\phi_{1} \rightarrow \phi_{2}\right) \in F$, then $\phi_{2} \in F$ (Modus Ponens)

$\left(R_{F}\right)$ If $\phi \in F$, then $\beta \phi \in F$ (rule of necessitation)

Modus Ponens requires that the agent is capable of making inferences on the set of propositions he has faith in. Together with $A_{0}$, it implies that the agent has faith in $\left(\phi_{1} \wedge \phi_{2}\right)$ if and only if he has faith in both propositions. 
The rule of necessitation states that the agent has faith in believing everything he has faith in, i.e., if the agent has faith in some proposition, then he has also faith in the fact that he believes this proposition.

Definition 1. The set $F$ of propositions the agent has faith in is the smallest set containing all propositions in $A$ which is closed under $R_{I}$ and $R_{F}$.

Formally, $A$ together with the rules $R_{I}$ and $R_{F}$ form a system of modal logic, as in Chellas (1980) or Fagin et al. (1995). Elements of $A$ are called axioms, while $R_{I}$ and $R_{F}$ are inference rules, and the elements of $F$ are called the theorems of the system of modal logic. The elements of $A$ and the inference rules that we use are standard in modal logic except for $A_{3}-A_{5}$ which are a weakening of the standard axioms: The benchmark Modal Logic system, which is called S5 and used to represent a logically omniscient agent, is defined by our set of axioms and inference rules, where $A_{3}-A_{5}$ are strengthened in that they are taken as axioms for every proposition, not just for primitive ones.

The following theorem shows that faith in introspection and the truth axiom for primitive propositions extends to the whole set of propositions.

Theorem 1. The agent has faith in the truth axiom and introspection for every proposition: For every $\phi \in \Phi$

$$
\begin{aligned}
& \text { 1. }(\beta \phi \rightarrow \phi) \in F \\
& \text { 2. }(\beta \phi \rightarrow \beta \beta \phi) \in F \\
& \text { 3. }(\neg \beta \phi \rightarrow \beta \neg \beta \phi) \in F
\end{aligned}
$$

The proof of Theorem 1 can be found in Appendix A.1.

A consequence of the previous result is that the system of modal logic defining $F$ is formally equivalent to the system S5 of a logically omniscient agent. Theorem 1 shows that, in $\mathrm{S} 5$, it is enough to assume the truth axiom and introspection on primitive propositions rather than on all propositions.

It is important to keep in mind that we do not take for granted that introspection holds for every proposition, or even for every primitive proposition. In that case, we would interpret elements of $F$ as properties that necessarily hold at every state of the world. In other words, we would assume that the agent is logically omniscient. Rather, we interpret $F$ as a set of propositions the agent has faith in, hence we have in mind an agent who has faith in his own logical omniscience. Whether or not he is right having faith in all 
propositions in $F$, i.e., whether all propositions in $F$ hold at a given state of the world, is a question studied in Section 4.2. This distinction will appear to be of considerable importance, in particular with respect to the question of unawareness.

\subsection{States of the world: Contingent beliefs}

The agent's beliefs at some state are of two types, faith - which consists of belief in propositions of $F$ - and contingent beliefs, which consist of beliefs about all other propositions. Faith is universal in the sense that belief in $F$ holds at every state. On the other hand, contingent belief is state-dependent. Consider for instance the primitive proposition $\phi$, which stands for "it is raining in New York". Obviously no element of $F$ can provide information to the agent about the truth value of $\phi$, and therefore the beliefs that the agent holds about $\phi$ depend on what he observes at every state, i.e., on whether he has credible information that it is raining. Hence, the truth value of $\beta \phi$ may differ across states.

Following Aumann (1999), a state $\omega$ assigns a truth value to every proposition in $\Phi$. A state thus provides a complete description of the facts (primitive propositions) and the agent's beliefs (epistemic propositions). It is a mapping from $\Phi$ to $\{0,1\}$, with the interpretation that $\phi$ is true at $\omega$ when $\omega(\phi)=1$ and false otherwise. We identify $\omega$ with the set of propositions that are true at $\omega$, and we write $\phi \in \omega$ when $\phi$ is true at $\omega$. Thus, we write $\omega=\{\phi \in \Phi: \omega(\phi)=1\}$. A state space is a collection of such states $\omega$. We restrict attention to states that satisfy the basic rules of logic, so we let $\Omega_{0}$ be the set of such mappings $\omega$, such that for every $\phi, \phi^{\prime} \in \Phi$ :

- $\phi \in \omega$, if and only if $\neg \phi \notin \omega$

- $\phi \wedge \phi^{\prime} \in \omega$, if and only if $\phi \in \omega$ and $\phi^{\prime} \in \omega$

- $\phi \vee \phi^{\prime} \in \omega$, if and only if $\phi \in \omega$ or $\phi^{\prime} \in \omega$

We have in mind an agent who is capable of reasoning and believes in all propositions of $F$.

Definition 2. Let $\Omega_{r}$ be the subset of $\Omega_{0}$ containing all states $\omega$ such that

$\left(A_{I}\right)\left(\beta\left(\phi_{1} \rightarrow \phi_{2}\right) \rightarrow\left(\beta \phi_{1} \rightarrow \beta \phi_{2}\right)\right) \in \omega$, for every $\phi_{1}, \phi_{2} \in \Phi$

$\left(A_{1}\right)\left(\beta\left(\phi_{1} \wedge \phi_{2}\right) \leftrightarrow\left(\beta \phi_{1} \wedge \beta \phi_{2}\right)\right) \in \omega$, for every $\phi_{1}, \phi_{2} \in \Phi$

$\left(A_{2}\right)\left(\left(\beta \phi_{1} \vee \beta \phi_{2}\right) \rightarrow \beta\left(\phi_{1} \vee \phi_{2}\right)\right) \in \omega$, for every $\phi_{1}, \phi_{2} \in \Phi$ 


\section{$\left(B_{F}\right) \beta \phi \in \omega$, for all $\phi \in F$}

The property $B_{F}$ states that the agent believes in all propositions in $F$, i.e., the agent's contingent beliefs cannot contradict the agent's universal beliefs. In other words, the belief in some element of $F$ is constant across states, e.g., the agent has faith in the fact that his beliefs are correct ${ }^{3}$, implying that he has faith in himself satisfying $A_{3}$, and therefore he believes in the proposition "it rains in New York whenever I believe so": $\beta(\beta \phi \rightarrow \phi) \in \omega$, for all $\omega \in \Omega_{r}$.

The restriction $B_{F}$ is quite reasonable, as the agent himself has proven - or assumed - these propositions, and therefore as long as he is confident that what he has assumed is true, he must also be confident that the conclusions he has reached are also true. However, the fact that the agent believes all propositions in $F$ does not necessarily mean that he is always right when doing so, i.e., he may wrongly believe some of the propositions he has faith to. Recall the example from the previous paragraph: The agent believes that every time he receives credible information about raining in New York, then this information is necessarily true. However, this need not be the case, as it would rule out the possibility that even though he believes his source, the information provided to him is wrong.

The wrong beliefs that the agent may have at some state are not arbitrary: The agent does not wrongly believe that he is capable of reasoning, i.e., we restrict our focus to states satisfying the main principles of belief, as expressed by $A_{1}, A_{2}$ and $A_{I}$.

A model in which the agent's faith is potentially delusional is very interesting from the bounded rationality point of view. It provides a framework in which all agents whether fully or boundedly rational - have faith in the same system of propositions $F$, and what distinguishes them is whether their assumptions on the world are satisfied or not. Bounded rationality is therefore contingent, and the reasoning processes of both the rational and boundedly rational agents are the same.

Situations of delusional faith are studied extensively in Section 5. We conclude this section by showing that delusional faith can only arise when the agent's assumptions $A_{3}-A_{5}$ on primitives are not satisfied.

Definition 3. Faith in $\phi$ is well founded at $\omega$ if $\phi \in F$ implies $\phi \in \omega$. Faith is well founded at $\omega$ if it is well founded for all propositions at $\omega$.

Theorem 2. Faith is well founded at $\omega \in \Omega_{r}$ if and only if $A_{3}-A_{5}$ are satisfied for all primitives at $\omega$.

\footnotetext{
${ }^{3}$ Feinberg (2004) imposes the same assumption, i.e., the agent (possibly wrongly) believes that he satisfies the truth axiom.
} 
The "only if" part of the theorem is obvious, as propositions in $A_{3}-A_{5}$ all belong to $F$, by definition. The "if" part shows that, if the agent's assumptions on his introspection capacities on primitives are correct, so are the logical conclusions he derives from them.

In particular, Theorem 2 shows that, whenever introspection holds on primitive propositions, it holds for every proposition. It provides a foundation for the introspection axioms, which are central in the literature (Samet, 1990), and allows to decompose these axioms into introspection for primitive propositions, which can be understood as the product of the agent's familiarity with these propositions, and the agent's deductive process, leading to introspection for all other propositions.

The subset $\Omega_{5}$ of states in $\Omega_{r}$ at which every proposition in $F$ holds, is the canonical state space for the modal logic system S5.

\section{Unawareness}

The aim of this section is to study situations where the agent has faith in his own reasoning ability, i.e., he correctly believes at all states that he has faith in $A_{0}-A_{5}$ and $A_{I}$, but he may wrongly believe some of the propositions in $F$ which are related to the truth axiom and introspection. Furthermore, we examine how these wrong beliefs are connected with the notions of unawareness and delusion.

\subsection{A state space with unawareness}

Unawareness about a phenomenon corresponds to a strong form of ignorance about this phenomenon, in the sense that the agent fails to recognize his own ignorance. Following Modica and Rustichini (1994, 1999), we define unawareness of $\phi$ as the conjunction of the ignorance of $\phi$ together with the ignorance of this ignorance: we let $u \phi$ stand for $\neg \beta \phi \wedge \neg \beta \neg \beta \phi$.

The definition for unawareness is relatively weak, in that $u \phi$ is compatible for instance with the agent believing that he does not believe that he does not believe believe $\phi$. Following Dekel et al. (1998), a stronger definition of unawareness requires the agent to disbelieve any proposition made by a sequence of "the agent believes" or "the agent does not believe" and ending in $\phi: \neg \beta \phi^{\prime}$ for all $\phi^{\prime} \in B(\phi)$, where $B(\phi)$ is the closure of $\{\beta \phi\}$ with respect to the operations $\neg$ and $\beta$. Our next result shows that, in every state where the agent has faith in every proposition in $F$, both definitions are equivalent: 
Proposition 1. Let $\omega \in \Omega_{r}$ be such that the agent believes every proposition that he has faith to: $\beta \phi \in \omega$ for every $\phi \in F$. For every proposition $\phi \in \Phi$, the agent is unaware of $\phi$ at $\omega$ if and only if $\neg \beta \phi^{\prime} \in \omega$ for all $\phi^{\prime} \in B(\phi)$.

Proposition 1 shows that in $\Omega_{r}$, failure of negative introspection on a proposition $\phi$ is necessarily accompanied by unawareness in the strong sense that the agent completely ignores $\phi$, e.g., the agent cannot be aware of a primitive $\phi$, while being unaware of his belief of $\phi$ : We cannot have $\neg u \phi$ and $u \beta \phi$ at the same $\omega$.

One advantage of using the weaker definition of unawareness is that $u \phi$ is a well defined proposition in $\Phi$. As shown by Proposition 1, it is equivalent to the stronger unawareness notion that is defined through the conjunction of an infinite family of propositions.

Since unawareness is a violation of negative introspection, we study states where negative introspection is relaxed. We consider states in which the agent believes in $F$, and in particular believes in negative introspection, but negative introspection may or may not hold. We also relax the truth axiom, since keeping the truth axiom would imply automatically that everything believed by the agent - including negative introspection holds. On the other hand, we assume positive introspection, which is not considered as a problematic axiom (Samet, 1990; Lipman, 1995).

Definition 4. Let $\Omega_{u}$ be the set of states in $\Omega_{r}$ in which positive introspection holds for every proposition.

In $\Omega_{u}$, the agent is capable of reasoning, believes in every proposition in $F$ and is capable of positive introspection. Compared to $\Omega_{5}$, negative introspection and the truth axiom do not necessarily hold in $\Omega_{u}$.

Now we relate unawareness to unawareness of primitive propositions:

Proposition 2. If the agent is unaware of some proposition at $\omega \in \Omega_{u}$ then he is unaware of some primitive proposition.

Proposition 2 shows that in $\Omega_{u}$, the only possible source of unawareness is unawareness of a primitive proposition.

The next result characterizes $F$ as the set of propositions that are universally believed in $\Omega_{u}$, i.e., the propositions $\phi$ such that $\beta \phi \in \omega$ for every $\omega \in \Omega_{u}$.

Proposition 3. $F$ is the set of propositions that are universally believed in $\Omega_{u}$. 


\subsection{Complexity of the state space}

As in e.g. Aumann (1999), we define the epistemic depth of a proposition $\phi$ as the number of nested belief operators found in this proposition. It is 0 for primitive propositions, the depth of $\neg \phi$ is the same as the depth of $\phi$, the depth of $\phi_{1} \vee \phi_{2}$ and $\phi_{1} \wedge \phi_{2}$ is the maximum of the depths of $\phi_{1}$ and $\phi_{2}$, and the depth of $\beta \phi$ is equal to the depth of $\phi$ plus one. Let $\Phi_{n}$ denote the set of propositions of epistemic depth at most $n$. Formally, we define $\Phi_{n}$ as the closure of the set $\left\{\phi, \beta \phi \mid \phi \in \Phi_{n-1}\right\}$ with respect to $\neg, \vee$ and $\wedge$.

As shown by Halpern (1995), states in $\Omega_{5}$ have an easy description. That is, two distinct states in $\Omega_{5}$ must differ in the truth value of the primitive propositions, or in the primitive beliefs. This is particularly interesting as what the agent believes about any proposition depends only on what he believes about the primitives, and therefore a state is determined by the primitive propositions and the primitive beliefs.

Proposition 4 (Halpern (1995)). Let $\omega, \omega^{\prime} \in \Omega_{5}$. If $\omega(\phi)=\omega^{\prime}(\phi)$ and $\omega(\beta \phi)=\omega^{\prime}(\beta \phi)$ for every $\phi \in \Phi_{0}$, then $\omega=\omega^{\prime}$.

Remark 1. Note that the values of the primitive propositions do not place any restrictions on the relationship among epistemic propositions, i.e., beliefs are determined inductively by beliefs (of lower or equal depth), and not by the truth values of the primitive. $\triangleleft$

The following example illustrates the relationship between primitive beliefs and states in $\Omega_{5}$.

Example 1. Suppose all propositions are derived from one primitive $\phi$, i.e., $\Phi_{0}=\{\phi, \neg \phi\}$. From Proposition 4 , it follows that the rational agent's state space is $\Omega_{5}=\left\{\omega_{1}, \ldots, \omega_{4}\right\}$ where:

$$
\begin{aligned}
& \omega_{1}=\{\phi, \beta \phi, \neg \beta \neg \phi, \ldots\}, \\
& \omega_{2}=\{\neg \phi, \neg \beta \phi, \beta \neg \phi, \ldots\}, \\
& \omega_{3}=\{\phi, \neg \beta \phi, \neg \beta \neg \phi, \ldots\}, \\
& \omega_{4}=\{\neg \phi, \neg \beta \phi, \neg \beta \neg \phi, \ldots\} .
\end{aligned}
$$

Why is the value of every proposition $\psi$ fixed at every $\omega \in\left\{\omega_{1}, \ldots, \omega_{4}\right\}$ ? We illustrate the mechanics underlying Proposition 4 for several such propositions.

First, note that the value of every such $\psi$ is determined by the value of primitives and the agent's beliefs. Remark also that the belief in any $\psi$ of the form $\psi=\psi_{1} \wedge \psi_{2}$, is equivalent to belief in both $\psi_{1}$ and $\psi_{2}$. 
How about belief in $\phi \vee \beta \phi$ ? Lemma 4 (in the appendix) together with $(\beta \phi \leftrightarrow \beta \beta \phi) \in \omega$ shows that for every $\omega \in \Omega_{5},(\beta(\phi \vee \beta \phi) \leftrightarrow \beta \phi) \in \omega$. Hence $\beta(\phi \vee \beta \phi)$ holds in $\omega_{1}$ only.

Lemma 4 also shows that $(\beta(\phi \vee \neg \beta \phi) \leftrightarrow(\beta \phi \vee \neg \beta \phi)) \in \omega$ for $\omega \in \Omega_{5}$, hence $\beta(\phi \vee \neg \beta \phi) \in \omega$ for all $\omega \in \Omega_{5}$.

Similarly $(\beta(\neg \phi \vee \beta \phi) \leftrightarrow(\beta \neg \phi \vee \beta \phi)) \in \omega$ for $\omega \in \Omega_{5}$. Thus $\beta(\neg \phi \vee \beta \phi)$ holds in $\omega_{1}$ and $\omega_{2}$, but not in $\omega_{3}$ or $\omega_{4}$.

More generally, it can be shown by induction that in $\Omega_{5}$, beliefs on propositions in $\Phi_{n}$ are determined by the belief on propositions in $\Phi_{n-1}$.

Next theorem shows that, in $\Omega_{u}$, beliefs are determined by the truth value of the primitives and the beliefs about every proposition of epistemic depth at most one.

Theorem 3. Let $\omega, \omega^{\prime} \in \Omega_{u}$. If $\omega(\phi)=\omega^{\prime}(\phi)$ and $\omega(\beta \phi)=\omega^{\prime}(\beta \phi)$ for every $\phi \in \Phi_{1}$, then $\omega=\omega^{\prime}$.

Remark 2. As in $\Omega_{5}$, beliefs are determined inductively by beliefs of lower or equal depth, and not by the primitives.

Theorem 3 shows that, although allowing for a very rich environment including possibilities of unawareness, the state space $\Omega_{u}$ still remains tractable. The driving force is that structure is provided through the faith system: Through a process of deductive reasoning, the agent is able to derive all higher order beliefs from beliefs about propositions of depth at most one.

In particular, Theorem 3 implies that $\Omega_{u}$ is finite if all propositions are constructed from an initial finite set of primitive propositions.

Example 2. Suppose as in Example 1 that all propositions are derived from a primitive $\phi$, i.e., $\Phi_{0}=\{\phi, \neg \phi\}$. In this case, $\Omega_{u}$ can be described as $\Omega_{u}=\left\{\omega_{1}^{+}, \ldots, \omega_{9}^{+}\right\} \cup\left\{\omega_{1}^{-}, \ldots, \omega_{9}^{-}\right\}$, where in $\phi$ holds in states $\omega_{i}^{+}, \neg \phi$ holds in states $\omega_{i}^{-}$, and all the agent's beliefs are the same in states $\omega_{i}^{+}$and $\omega_{i}^{-}$. An agent's "state of mind", which is the same in $\omega_{i}^{+}$and $\omega_{i}^{-}$, can be written as $\tilde{\omega}_{i}=\omega_{i}^{+} \cap \omega_{i}^{-}$. It is straightforward that both $\omega_{i}^{+}$and $\omega_{i}^{-}$are known once $\tilde{\omega}_{i}$ is, so that the description of the state space can be completed by the description of the agent's "states of mind" $\tilde{\omega}_{1}, \ldots, \tilde{\omega}_{9}$. 
- States of mind in which the agent is aware of $\phi$ and $\neg \phi$

$$
\begin{aligned}
& \tilde{\omega}_{1}=\{\beta \phi, \neg \beta \neg \phi, \beta \beta \phi, \beta \neg \beta \neg \phi, \beta(\phi \vee \beta \neg \phi), \beta(\neg \phi \vee \beta \phi), \ldots\} \\
& \tilde{\omega}_{2}=\{\neg \beta \phi, \beta \neg \phi, \beta \neg \beta \phi, \beta \beta \neg \phi, \beta(\phi \vee \beta \neg \phi), \beta(\neg \phi \vee \beta \phi), \ldots\} \\
& \tilde{\omega}_{3}=\{\neg \beta \phi, \neg \beta \neg \phi, \beta \neg \beta \phi, \beta \neg \beta \neg \phi, \neg \beta(\phi \vee \beta \neg \phi), \neg \beta(\neg \phi \vee \beta \phi), \ldots\}
\end{aligned}
$$

States $\left\{\omega_{1}^{+}, \omega_{2}^{-}, \omega_{3}^{+}, \omega_{3}^{-}\right\}$coincide with $\omega_{1}, \ldots, \omega_{4} \in \Omega_{5}$. In states $\omega_{1}^{-}$and $\omega_{2}^{+}$, the agent exhibits delusion, since either $\phi$ or $\neg \phi$ is believed but doesn't hold.

- States of mind in which the agent is unaware of $\neg \phi$ and is aware of $\phi$ :

$$
\tilde{\omega}_{4}=\{\neg \beta \phi, \neg \beta \neg \phi, \beta \neg \beta \phi, \neg \beta \neg \beta \neg \phi, \neg \beta(\phi \vee \beta \neg \phi), \neg \beta(\neg \phi \vee \beta \phi), \ldots\}
$$

The fact that $\neg \beta(\neg \phi \vee \beta \phi) \in \tilde{\omega}_{4}$ is straightforward: Suppose otherwise. Then, $\beta(\neg \beta \phi \rightarrow \neg \phi) \in \tilde{\omega}_{4}$ contradicts $\neg \beta \phi \in \tilde{\omega}_{4}$, because of $A_{I}$. The fact that $\neg \beta(\phi \vee$ $\beta \neg \phi) \in \tilde{\omega}_{4}$ also follows by contradiction: Otherwise, it follows from $A_{4}$ and $A_{I}$ that $\beta(\beta \neg \beta \neg \phi \rightarrow \beta \phi) \in \tilde{\omega}_{4}$. Then, it follows from $A_{0}$ that $\beta(\neg \beta \phi \rightarrow \neg \beta \neg \beta \neg \phi) \in \tilde{\omega}_{4}$, and again from $A_{I}$ it follows $(\beta \neg \beta \phi \rightarrow \beta \neg \beta \neg \beta \neg \phi) \in \tilde{\omega}_{4}$, which contradicts the unawareness of $\neg \phi$.

- States of mind in which the agent is unaware of $\phi$ and is aware of $\neg \phi$ :

$$
\tilde{\omega}_{5}=\{\neg \beta \phi, \neg \beta \neg \phi, \neg \beta \neg \beta \phi, \beta \neg \beta \neg \phi, \neg \beta(\phi \vee \beta \neg \phi), \neg \beta(\neg \phi \vee \beta \phi), \ldots\}
$$

The arguments regarding the beliefs about $\phi \vee \beta \neg \phi$ and $\neg \phi \vee \beta \phi$ at $\tilde{\omega}_{5}$ are the same as in $\tilde{\omega}_{4}$.

- States of mind in which the agent is unaware of both $\phi$ and $\neg \phi$ :

$$
\begin{aligned}
& \tilde{\omega}_{6}=\{\neg \beta \phi, \neg \beta \neg \beta \phi, \neg \beta \neg \phi, \neg \beta \neg \beta \neg \phi, \beta(\phi \vee \beta \neg \phi), \beta(\neg \phi \vee \beta \phi), \ldots\} \\
& \tilde{\omega}_{7}=\{\neg \beta \phi, \neg \beta \neg \beta \phi, \neg \beta \neg \phi, \neg \beta \neg \beta \neg \phi, \neg \beta(\phi \vee \beta \neg \phi), \beta(\neg \phi \vee \beta \phi), \ldots\} \\
& \tilde{\omega}_{8}=\{\neg \beta \phi, \neg \beta \neg \beta \phi, \neg \beta \neg \phi, \neg \beta \neg \beta \neg \phi, \beta(\phi \vee \beta \neg \phi), \neg \beta(\neg \phi \vee \beta \phi), \ldots\} \\
& \tilde{\omega}_{9}=\{\neg \beta \phi, \neg \beta \neg \beta \phi, \neg \beta \neg \phi, \neg \beta \neg \beta \neg \phi, \neg \beta(\phi \vee \beta \neg \phi), \neg \beta(\neg \phi \vee \beta \phi), \ldots\}
\end{aligned}
$$

Simultaneous unawareness of both $\phi$ and $\neg \phi$ allows any beliefs for $\phi \vee \beta \neg \phi$ and $\neg \phi \vee \beta \phi$, e.g., $\tilde{\omega}_{6}$ and $\tilde{\omega}_{7}$ differ in the beliefs of $\phi \vee \beta \neg \phi$, implying that primitive 
beliefs and awareness about the primitives do not suffice to characterize a state. Instead, some more information is needed (see Theorem 4 below).

Two things become clear from this example, which provide an illustration of our previous results: First, whenever the agent's beliefs do not coincide with beliefs in $\Omega_{5}$, the agent is unaware (see Proposition 2) or delusional of at least one primitive, and second, unlike in $\Omega_{5}$, primitive beliefs alone do not suffice for characterizing the agent's state of mind in $\Omega_{u}$ (see Theorem 3).

Finally, note that imposing $(u \phi \leftrightarrow u \neg \phi) \in \omega$, like Modica and Rustichini (1994), would eliminate the states of mind $\tilde{\omega}_{4}$ and $\tilde{\omega}_{5}$, but not the other states. In particular, our model is compatible with such a restriction, even if, for the sake of generality, we do not impose it.

Now we present an alternative description of states in $\Omega_{u}$ in which a state is described through primitive and beliefs on primitives as well as agent's awareness of propositions of depth at most one.

Theorem 4. Let $\omega, \omega^{\prime} \in \Omega_{u}$. If $\omega(\phi)=\omega^{\prime}(\phi)$ and $\omega(\beta \phi)=\omega^{\prime}(\beta \phi)$ for every $\phi \in \Phi_{0}$, and $\omega(u \phi)=\omega^{\prime}(u \phi)$ for all $\phi \in \Phi_{1}$, then $\omega=\omega^{\prime}$.

This last theorem has a natural appeal: In order to describe the agent's beliefs, it is enough to describe the agent's beliefs on primitive propositions, as well as the set of propositions the agent is aware of. If at some states $\omega$ and $\omega^{\prime}$, the agent is aware of the same propositions and has the same beliefs on primitive propositions, then the agent's beliefs on every proposition is the same at $\omega$ and at $\omega^{\prime}$. Furthermore, the theorem shows it is enough to restrict attention to awareness of propositions of depth at most 1: All beliefs are fully described by beliefs on primitive and awareness of propositions of depth at most one. In particular, two states in which the truth value of primitives, the agent's beliefs on primitives, and the awareness of propositions of depth one are the same, coincide.

\section{Semantics}

The state space models introduced in Sections 4 and 5 are syntactic: Each state corresponds to a truth assignment for every proposition, including the agent's belief. Semantic models offer an alternative representation of the agent's beliefs.

Formally, a semantic model is a tuple $(\Omega, P)$, where $\Omega$ is the state space and $P: \Omega \rightarrow 2^{\Omega}$ denotes the agent's possibility correspondence: at state $\Omega, P(\omega)$ is the set of states that the agent considers as possible. 
It is common to define a belief operator $B$ from the possibility correspondence by the relation:

$$
B E_{\phi}:=\left\{\omega \in \Omega: P(\omega) \subseteq E_{\phi}\right\}
$$

where $E_{\phi}:=\left\{\omega^{\prime} \in \Omega: \phi \in \omega^{\prime}\right\}$. The relation $\omega \in B E_{\phi}$ reads "the agent (semantically) believes $\phi$ at $\omega$ ", and holds whenever $\phi$ is true at all states in $P(\omega)$.

Each syntactic model has a natural semantic counterpart: On a syntactic state space $\Omega$, define a possibility correspondence $P$ by

$$
P(\omega):=\left\{\omega^{\prime} \in \Omega: b(\omega) \subseteq \omega^{\prime}\right\}
$$

where $b(\omega):=\{\phi \in \Phi: \beta \phi \in \omega\}$.

Aumann (1999) shows that $\Omega_{5}$ has a semantic representation, i.e., with the possibility correspondence $P$ and the semantic belief operator $B$ defined as above, the syntactic and the semantic beliefs coincide at all states in $\Omega_{5}$. Formally, $B E_{\phi}=E_{\beta \phi}$ for every proposition $\phi$. This result is very useful, as it allows to work equivalently using either the semantic or the syntactic model, and semantic models can often be manipulated more easily than syntactic ones.

We extend Aumann's result to $\Omega_{u}$. In order to do so, we rely on the possibility correspondence $P$ defined as above, but use a different definition of semantic beliefs. Let $A E_{\phi}:=\left\{\omega \in \Omega_{u}: \neg u \phi \in \omega\right\}$ be the set of states at which the agent is aware of $\phi$, and $B E_{\phi}:=\left\{\omega \in \Omega: P(\omega) \subseteq E_{\phi}\right\}$ as above. We define the belief operator $B_{u}$ by:

$$
B_{u} E_{\phi}:=A E_{\phi} \cap B E_{\phi}
$$

According to this definition, in order for the agent to (semantically) believe $\phi$, it does not suffice that $\phi$ holds everywhere in $P(\omega)$. We also require that the agent is aware of $\phi$. This requirement is aligned with the idea of implicit and explicit belief, introduced by Fagin and Halpern (1988), which is further discussed in Section 7.1.

The following result formalizes the equivalence between the syntactic beliefs in $\Omega_{u}$, and semantic beliefs induced by $B_{u}$.

Theorem 5. $B_{u} E_{\phi}=E_{\beta \phi}$.

The following result shows that our definition of $B_{u}$ is indeed semantic, i.e., $B_{u} E$ does not depend on the particular proposition $\phi$ such that $E=E_{\phi}$.

Proposition 5. If $E_{\phi_{1}}=E_{\phi_{2}}$, then $B_{u} E_{\phi_{1}}=B_{u} E_{\phi_{2}}$. 
Note that if the set of primitive propositions is finite, then every subset of $\Omega_{u}$ corresponds to some proposition in $\Phi$, and therefore $B_{u} E$ is defined for every subset $E$ of $\Omega_{u}$.

The following consequence of Theorem 5 shows that if a proposition is true in every state of $\Omega_{u}$, i.e., it is a tautology of $\Omega_{u}$, the agent believes it in every state of $\Omega_{u}$.

Corollary 1. $B_{u} \Omega_{u}=\Omega_{u}$.

To prove the Corollary, observe that tautologies of the form $\beta \phi, \phi \in F$, are believed at every state in $\Omega_{u}$. Proposition 5 shows that this is actually the case of every tautology, i.e., if $\phi$ is a tautology of $\Omega_{u}$, it is believed by the agent at every state of $\Omega_{u}$.

The Corollary has the desirable, natural implication that $\Omega_{u}$ is a complete representation of the modal logic system $F$. Furthermore, since $F$ coincides with S5 (see Theorem 1 ), one obtains the result that propositions in S5 are believed at every state of $\Omega_{u}$ :

Corollary 2. $B_{u} \Omega_{5}=\Omega_{u}$.

This last corollary emphasizes the fact that, at all states in $\Omega_{u}$, including those not belonging to $\Omega_{5}$, the agent perceives $\Omega_{5}$ as the "actual" state space. This is in line with the main idea of the paper that the agent reasons "as if" the state space was $\Omega_{5}$, even in states outside $\Omega_{5}$. We further discuss this point in Section 7.5.

\section{Discussion}

\subsection{Explicit and implicit beliefs}

The notion of unawareness was first formalized, in the context of Modal Logic, by Fagin and Halpern (1988). Their paper introduces separate modalities for explicit belief - which is equivalent to the standard notion of belief - and for implicit belief, which can be thought as the set of logical consequences of the explicitly believed propositions. A proposition is explicitly believed whenever the agent implicitly believes it and is aware of it. The relationship to our model becomes more transparent when one looks at the semantic representation in Section 6. In this semantic model, implicit belief corresponds to the usual semantic belief operator: $\phi$ is implicitly believed at $\omega$ when $P(\omega) \subseteq E_{\phi}$. Our notion of belief in the semantic model requires both implicit belief and awareness, it is therefore a notion of explicit belief. Since belief in the syntactic model is equivalent to implicit belief and awareness in the semantic model, it is also to be thought of as explicit belief. 


\subsection{Reasoning, unawareness and knowledge}

Modica and Rustichini (1994) show that, when modeling knowledge (i.e., assuming the that every proposition believed by the agent holds) and positive introspection holds, then negative introspection is equivalent to the symmetry axiom ${ }^{4}$, implying that unawareness cannot be modeled using knowledge unless either symmetry or reasoning is relaxed. Although in their followup paper (Modica and Rustichini, 1999) they acknowledge the desirability of a unified reasoning process by mentioning that "it is not at all the case that a subject who is aware of fewer things than another must necessarily be less capable of logical reasoning than the latter", they defend the idea that the agent's reasoning must be relaxed in order to model unawareness.

Following Modica and Rustichini (1999), a strand of literature (Heifetz et al., 2006, 2008; Li, 2009) studies models of unawareness in which the agent can reason only about the propositions he is aware of.

Our model departs from the above mentioned literature in that we consider (confident) belief, instead of knowledge. Modeling unawareness in a belief model was already pointed out by Modica and Rustichini (1999) as in important question. To quote them:

Knowledge excludes the possibility that the agent "knows" or, better, "believes", something which is false. This distinction opens a question: the one of defining and analysing awareness with belief, rather than knowledge. This would consist in dropping the truth axiom from our system. This is an important question, that we do not discuss here.

\subsection{Canonical states}

Our approach follows that of Aumann (1999) and Samet (1990) in that a state assigns a truth value to all propositions, including those concerning the agent's beliefs about his own beliefs, and so on. Several papers - including, for instance, Modica and Rustichini (1994), Halpern (2001) and Heifetz et al. (2006, 2008) - restrict their study to so called "canonical states" by making the additional assumption that two states that coincide on primitives and on the agent's beliefs on primitives necessarily coincide. As shown by Proposition 4 , this is without loss in generality when considering states in $\Omega_{5}$. On the other hand, distinct states on $\Omega_{u}$ may agree on primitives and on the agent's beliefs on primitives, as these do not suffice to describe a state in $\Omega_{u}$. This can be see for instance in Example 2.

\footnotetext{
${ }^{4}$ According to the symmetry axiom, the agent is aware of $\phi$ if and only if he is aware of $\neg \phi$.
} 
Theorems 4 shows, on the other hand, that beliefs of propositions of high epistemic order are unnecessary to describe a state, as two states that coincide on primitives and on beliefs of propositions of epistemic depth at most one necessarily coincide. This shows that, in the state space with unawareness, the appropriate notion of a canonical state is a description of primitives together with beliefs of propositions of epistemic depth at most one.

\subsection{Awareness properties}

Several properties of awareness have been previously introduced and studied, and are considered by some authors as desiderata for an awareness notion. The model introduced by (Heifetz et al., 2006, 2008) is constructed in order to fulfill these properties. Our approach is more agnostic on the properties of unawareness, and tries to derive these properties from a natural representation of the agent's mind rather than imposing them. Note however that, as shown by Example 2, it is always possible to restrict attention to subsets of $\Omega_{u}$ where some extra structure on awareness (such as symmetry, for instance) is imposed.

\subsection{States and perceived states}

In our model there exist states (from the modeler's point of view) in which the agent is unaware of some primitive propositions, but the agent does not consider such states as being possible. The distinction between the states deemed as possible by the agent and those which may actually arise is not new in the literature. For instance, Geanakoplos (1989) shows that apparent failures of rational information processing can be explained if the agent considers as possible states of the world that the agent ignores. More recently, in their fundamental work showing some of the main difficulties arising when modeling unawareness, Dekel et al. (1998) show that this distinction is necessary in order to capture any meaningful notion of unawareness.

The distinction between the states of the world arising and those considered as possible by the agent can be best seen in the semantic model of Section 6. States belong to two categories. The states in $\Omega_{5}$ are the states in which the agent is aware of every proposition. As in Bacharach (1985) and Samet (1990), the agent's possibility correspondence defines a partition of the states in $\Omega_{5}$. The agent does not consider states outside of $\Omega_{5}$ as being possible. At such states, the only states the agent considers as possible belong to $\Omega_{5}$, 
hence the agent exhibits delusion.

\subsection{Extensions}

Our model explicitly distinguishes beliefs arising from two different sources: Universal beliefs, which follow from faith, and contingent beliefs, which follow from observation at every instance. We show that this distinction provides a natural rationale for unawareness and delusion, while maintaining the agent's reasoning process.

Alternative models can be obtained under variations of the faith system, or under different assumption on contingent beliefs. The exploration of these variations can provide a fruitful direction for future research.

\subsection{Final remarks}

Unawareness has been a recognized phenomenon for several decades, and has formed a long standing modeling puzzle in formal epistemology. Its importance in Economics can be exemplified by recent work (see, e.g., Feinberg (2004) and Heifetz et al. (2009)) showing that unawareness allows to capture the agent's behaviors in a way that differs significantly from the classical framework of incomplete information à la Harsanyi (196768). The model introduced in this paper provides a model of unawareness which is both simple and has intuitive appeal. We hope that, by enhancing our understanding the formation of agent's beliefs, and by offering a tractable model, it will contribute to our comprehension of the role of unawareness in economic contexts.

\section{A Appendix}

\section{A.1 Proofs of Section 4}

Definition 5. Let $\left(\phi_{1} \stackrel{F}{\rightarrow} \phi_{2}\right)$ be a shorthand for the following statement:

$$
\text { if } \phi_{1} \in F \text { then } \phi_{2} \in F \text {. }
$$

Lemma 1. For $\phi_{1}, \phi_{2}, \phi_{3}, \phi_{4} \in \Phi$ :

1. $\left(\phi_{1} \rightarrow \phi_{2}\right) \stackrel{F}{\rightarrow}\left(\neg \phi_{2} \rightarrow \neg \phi_{1}\right)$,

2. $\left(\left(\phi_{1} \rightarrow \phi_{2}\right) \wedge\left(\phi_{2} \rightarrow \phi_{3}\right)\right) \stackrel{F}{\rightarrow}\left(\phi_{1} \rightarrow \phi_{3}\right)$, 
3. If $\phi_{1} \stackrel{F}{\rightarrow} \phi_{3}$ and $\phi_{2} \stackrel{F}{\rightarrow} \phi_{4}$, then $\left(\phi_{1} \wedge \phi_{2}\right) \stackrel{F}{\rightarrow}\left(\phi_{3} \wedge \phi_{4}\right)$.

Proof. 1. It follows directly from the definition of the implication.

2. Consider the following sequence of tautologies:

$$
\begin{aligned}
\left.\left(\left(\phi_{1} \rightarrow \phi_{2}\right) \wedge\left(\phi_{2} \rightarrow \phi_{3}\right)\right)\right) & \stackrel{\mathrm{F}}{\rightarrow}\left(\left(\neg \phi_{1} \wedge \neg \phi_{2}\right) \vee\left(\neg \phi_{1} \wedge \phi_{3}\right) \vee\left(\phi_{2} \wedge \neg \phi_{2}\right) \vee\left(\phi_{2} \wedge \phi_{3}\right)\right) \\
& \stackrel{\mathrm{F}}{\rightarrow}\left(\left(\neg \phi_{1} \wedge \neg \phi_{2}\right) \vee\left(\neg \phi_{1} \wedge \phi_{3}\right) \vee\left(\phi_{2} \wedge \phi_{3}\right)\right) \\
& \stackrel{\mathrm{F}}{\rightarrow}\left(\neg \phi_{1} \vee \phi_{3}\right) \\
& \stackrel{\mathrm{F}}{\rightarrow}\left(\phi_{1} \rightarrow \phi_{3}\right) .
\end{aligned}
$$

3. The following relationships hold:

$$
\begin{aligned}
& \left(\phi_{1} \wedge \phi_{2}\right) \stackrel{\mathrm{F}}{\rightarrow} \phi_{1} \stackrel{\mathrm{F}}{\rightarrow} \phi_{3}, \\
& \left(\phi_{1} \wedge \phi_{2}\right) \stackrel{\mathrm{F}}{\rightarrow} \phi_{2} \stackrel{\mathrm{F}}{\rightarrow} \phi_{4} .
\end{aligned}
$$

That is, if $\left(\phi_{1} \wedge \phi_{2}\right) \in F$ then $\left(\phi_{3} \wedge \phi_{4}\right) \in F$.

Lemma 2. For some $\phi \in \Phi$, let the agent have faith in the truth axiom and introspection for $\phi$ and $\neg \phi$. Then, the agent has faith in the truth axiom and introspection for $\beta \phi$ and $\neg \beta \phi$.

Proof. Truth Axiom: It follows by hypothesis that $(\beta \phi \rightarrow \phi) \in F$. Thus,

$$
(\beta \phi \rightarrow \phi) \stackrel{\left(\text { by } A_{I}\right)}{\stackrel{\mathrm{F}}{\rightarrow}}(\beta \beta \phi \rightarrow \beta \phi) .
$$

It follows from $A_{3}$ that $(\beta \phi \rightarrow \beta \beta \phi) \in F$. Thus,

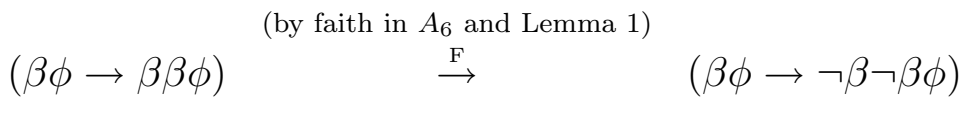

$$
\begin{aligned}
& \stackrel{\mathrm{F}}{\rightarrow} \quad(\beta \neg \beta \phi \rightarrow \neg \beta \phi) .
\end{aligned}
$$

Positive introspection: It follows by hypothesis that $(\beta \phi \rightarrow \beta \beta \phi) \in F$ and $(\neg \beta \phi \rightarrow$ $\beta \neg \beta \phi) \in F$. Thus,

$$
(\beta \phi \rightarrow \beta \beta \phi) \stackrel{\mathrm{F}}{\rightarrow}(\beta \beta \phi \rightarrow \beta \beta \beta \phi),
$$

and

$$
(\neg \beta \phi \rightarrow \beta \neg \beta \phi) \stackrel{\text { by } \left.A_{I}\right)}{\stackrel{\mathrm{F}}{\rightarrow}}(\beta \neg \beta \phi \rightarrow \beta \beta \neg \beta \phi) .
$$


Negative introspection: It follows by hypothesis that $(\beta \phi \rightarrow \beta \beta \phi) \in F$. Thus,

$$
\begin{aligned}
& (\beta \phi \rightarrow \beta \beta \phi) \quad \stackrel{\text { (by Lemma 1) }}{\stackrel{\mathrm{F}}{\rightarrow}} \quad(\neg \beta \beta \phi \rightarrow \neg \beta \phi) \\
& \text { (by faith in } A_{5} \text { and Lemma 1) } \\
& \stackrel{\mathrm{F}}{\rightarrow} \quad(\neg \beta \beta \phi \rightarrow \beta \neg \beta \phi) \\
& \text { (by faith in } A_{3} \text { and } R_{I} \text { ) } \\
& \stackrel{\mathrm{F}}{\rightarrow} \quad(\neg \beta \beta \phi \rightarrow \beta \neg \beta \phi) \wedge(\beta \neg \beta \phi \rightarrow \beta \neg \beta \beta \phi) \\
& \text { (by Lemma 1) } \\
& \stackrel{\mathrm{F}}{\rightarrow} \quad(\neg \beta \beta \phi \rightarrow \beta \neg \beta \beta \phi) .
\end{aligned}
$$

It follows by hypothesis that $(\neg \beta \phi \rightarrow \beta \neg \beta \phi) \in F$. Thus,

$$
\begin{aligned}
& (\neg \beta \phi \rightarrow \beta \neg \beta \phi) \quad \stackrel{\text { (by Lemma 1) }}{\rightarrow} \quad(\neg \beta \neg \beta \phi \rightarrow \beta \phi) \\
& \text { (by faith in } \left.A_{4} \text { and Lemma } 1\right) \quad(\stackrel{\mathrm{F}}{\rightarrow} \quad(\neg \beta \neg \beta \phi \rightarrow \beta \beta \phi) \\
& \text { (by faith in } A_{4} \text { and Lemma } 1 \text { ) } \\
& \stackrel{\mathrm{F}}{\rightarrow} \quad(\neg \beta \neg \beta \phi \rightarrow \beta \beta \beta \phi) \\
& \text { (by faith in } A_{6} \text { and } A_{I} \text { ) } \\
& \stackrel{\mathrm{F}}{\rightarrow} \quad(\neg \beta \neg \beta \phi \rightarrow \beta \beta \beta \phi) \wedge(\beta \beta \beta \phi \rightarrow \beta \neg \beta \neg \beta \phi) \\
& \text { (by Lemma 1) } \\
& \stackrel{\mathrm{F}}{\rightarrow} \quad(\neg \beta \neg \beta \phi \rightarrow \beta \neg \beta \neg \beta \phi),
\end{aligned}
$$

which completes the proof.

Lemma 3. Let the agent have faith in the truth axiom and introspection for $\phi_{1}$ and $\phi_{2}$.

Then, the agent has faith in the truth axiom and introspection for $\phi_{1} \wedge \phi_{2}$.

Proof. Truth Axiom: It follows from $A_{1}$ that $\left(\beta\left(\phi_{1} \wedge \phi_{2}\right) \rightarrow\left(\beta \phi_{1} \wedge \beta \phi_{2}\right)\right) \in F$. Then,

$$
\left(\beta\left(\phi_{1} \wedge \phi_{2}\right) \rightarrow\left(\beta \phi_{1} \wedge \beta \phi_{2}\right)\right) \quad \stackrel{\text { (by faith in } \left.A_{3}\right)}{\stackrel{\mathrm{F}}{\rightarrow}}\left(\beta\left(\phi_{1} \wedge \phi_{2}\right) \rightarrow\left(\phi_{1} \wedge \phi_{2}\right)\right)
$$

Positive introspection: Likewise,

$$
\begin{array}{ll} 
& \left(\beta\left(\phi_{1} \wedge \phi_{2}\right) \rightarrow\left(\beta \phi_{1} \wedge \beta \phi_{2}\right)\right) \\
\stackrel{\text { (by faith in } \left.A_{4}\right)}{\stackrel{\mathrm{F}}{\rightarrow}} & \left(\beta\left(\phi_{1} \wedge \phi_{2}\right) \rightarrow\left(\beta \phi_{1} \wedge \beta \phi_{2}\right)\right) \wedge\left(\left(\beta \phi_{1} \wedge \beta \phi_{2}\right) \rightarrow\left(\beta \beta \phi_{1} \wedge \beta \beta \phi_{2}\right)\right) \\
\stackrel{\mathrm{F}}{\rightarrow} & \left(\beta\left(\phi_{1} \wedge \phi_{2}\right) \rightarrow \beta \beta\left(\phi_{1} \wedge \phi_{2}\right)\right) .
\end{array}
$$

Negative introspection: It follows from $A_{1}$ and Lemma 1 that $\left(\neg \beta\left(\phi_{1} \wedge \phi_{2}\right) \rightarrow\right.$ 
$\left.\left(\neg \beta \phi_{1} \vee \neg \beta \phi_{2}\right)\right) \in F$. Thus,

$$
\left(\neg \beta\left(\phi_{1} \wedge \phi_{2}\right) \rightarrow\left(\neg \beta \phi_{1} \vee \neg \beta \phi_{2}\right)\right) \quad \stackrel{\text { (by faith in } \left.A_{5}\right)}{\stackrel{\mathrm{F}}{\rightarrow}} \quad\left(\neg \beta\left(\phi_{1} \wedge \phi_{2}\right) \rightarrow \beta \neg \beta\left(\phi_{1} \wedge \phi_{2}\right)\right),
$$

which completes the proof.

Lemma 4. Let the agent have faith in introspection for $\phi_{1}$ and $\phi_{2}$. Then, $\left(\beta\left(\beta \phi_{1} \vee \phi_{2}\right) \leftrightarrow\right.$ $\left.\left(\beta \phi_{1} \vee \beta \phi_{2}\right)\right) \in F$.

Proof. It follows from $\left(\beta \phi_{1} \rightarrow \beta \beta \phi_{1}\right) \in F$ that $\left(\left(\beta \phi_{1} \vee \beta \phi_{2}\right) \rightarrow\left(\beta \beta \phi_{1} \vee \beta \phi_{2}\right)\right) \in F$. Thus, it follows from $A_{2}$ and Lemma 1 that

$$
\left(\left(\beta \phi_{1} \vee \beta \phi_{2}\right) \rightarrow \beta\left(\beta \phi_{1} \vee \phi_{2}\right)\right) \in F
$$

For the converse, it follows by definition that $\left(\beta\left(\beta \phi_{1} \vee \phi_{2}\right) \rightarrow \beta\left(\neg \beta \phi_{1} \rightarrow \phi_{2}\right)\right) \in F$. Thus,

$$
\begin{aligned}
& \left(\beta\left(\beta \phi_{1} \vee \phi_{2}\right) \rightarrow \beta\left(\neg \beta \phi_{1} \rightarrow \phi_{2}\right)\right) \\
& \text { (by definition) } \\
& \stackrel{\mathrm{F}}{\rightarrow} \quad\left(\neg \beta\left(\beta \phi_{1} \vee \phi_{2}\right) \vee \beta\left(\neg \beta \phi_{1} \rightarrow \phi_{2}\right)\right. \\
& \stackrel{\left(\text { by } A_{I}\right)}{\stackrel{\mathrm{F}}{\rightarrow}} \quad\left(\neg \beta\left(\beta \phi_{1} \vee \phi_{2}\right) \vee\left(\beta \neg \beta \phi_{1} \rightarrow \beta \phi_{2}\right)\right) \\
& \text { (by definition) } \\
& \stackrel{\mathrm{F}}{\rightarrow} \quad\left(\beta\left(\beta \phi_{1} \vee \phi_{2}\right) \rightarrow\left(\neg \beta \neg \beta \phi_{1} \vee \beta \phi_{2}\right)\right) \\
& \text { (by faith in } A_{4} \text { and Lemma 1) } \\
& \stackrel{\mathrm{F}}{\rightarrow} \quad\left(\beta\left(\beta \phi_{1} \vee \phi_{2}\right) \rightarrow\left(\beta \phi_{1} \vee \beta \phi_{2}\right)\right),
\end{aligned}
$$

which completes the proof.

Lemma 5. Let the agent have faith in the truth axiom and introspection for $\phi_{1}$ and $\phi_{2}$.

Then, the agent has faith in the truth axiom and introspection for

1. $\beta \phi_{1} \vee \phi_{2}$, and

2. $\neg \beta \phi_{1} \vee \phi_{2}$.

Proof. 1. It follows from Lemma 4 that $\left(\beta\left(\beta \phi_{1} \vee \phi_{2}\right) \rightarrow\left(\beta \phi_{1} \vee \beta \phi_{2}\right)\right) \in F$.

TRUTH AXIOM: Thus,

$$
\left(\beta\left(\beta \phi_{1} \vee \phi_{2}\right) \rightarrow\left(\beta \phi_{1} \vee \beta \phi_{2}\right)\right) \stackrel{\text { (by faith in } A_{3} \text { and Lemma 1) }}{\stackrel{\mathrm{F}}{\rightarrow}} \quad\left(\beta\left(\beta \phi_{1} \vee \phi_{2}\right) \rightarrow\left(\beta \phi_{1} \vee \phi_{2}\right)\right)
$$


Positive introspection: Thus,

$$
\begin{array}{cl} 
& \left(\beta\left(\beta \phi_{1} \vee \phi_{2}\right) \rightarrow\left(\beta \phi_{1} \vee \beta \phi_{2}\right)\right) \\
\stackrel{\stackrel{\mathrm{F}}{\rightarrow}}{\left(\text { by faith in } A_{4}\right. \text { and Lemma 1) }} & \left(\beta\left(\beta \phi_{1} \vee \phi_{2}\right) \rightarrow\left(\beta \beta \beta \phi_{1} \vee \beta \beta \phi_{2}\right)\right) \\
\stackrel{\mathrm{F}}{\rightarrow} & \left(\beta\left(\beta \phi_{1} \vee \phi_{2}\right) \rightarrow \beta \beta\left(\beta \phi_{1} \vee \phi_{2}\right)\right) .
\end{array}
$$

Negative introspection: It follows from Lemma 1 that $\left(\neg \beta\left(\beta \phi_{1} \vee \phi_{2}\right) \rightarrow \neg\left(\beta \phi_{1} \vee\right.\right.$ $\left.\left.\beta \phi_{2}\right)\right) \in F$. Thus,

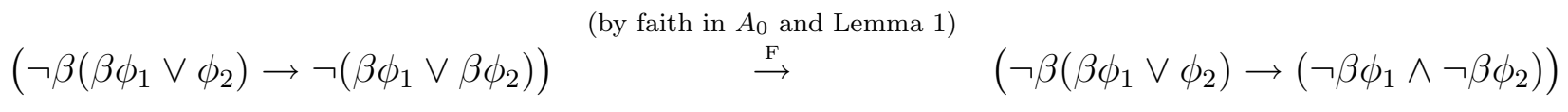

$$
\begin{aligned}
& \stackrel{\text { (by faith in } A_{5} \text { and Lemma 1) }}{\stackrel{\mathrm{F}}{\rightarrow}} \quad\left(\neg \beta\left(\beta \phi_{1} \vee \phi_{2}\right) \rightarrow\left(\beta \neg \beta \phi_{1} \wedge \beta \neg \beta \phi_{2}\right)\right) \\
& \stackrel{\text { (by Lemmas } 1 \text { and } 3)}{\stackrel{F}{\rightarrow}} \quad\left(\neg \beta\left(\beta \phi_{1} \vee \phi_{2}\right) \rightarrow \beta \neg\left(\beta \phi_{1} \vee \beta \phi_{2}\right)\right) \\
& \stackrel{\text { (by Lemma } 4)}{\stackrel{\mathrm{F}}{\rightarrow}} \quad\left(\neg \beta\left(\beta \phi_{1} \vee \phi_{2}\right) \rightarrow \beta \neg \beta\left(\beta \phi_{1} \vee \phi_{2}\right)\right) .
\end{aligned}
$$

2. It follows from faith in $A_{5}$ and Lemma 2 that $\left(\left(\neg \beta \phi_{1} \vee \phi_{2}\right) \leftrightarrow\left(\beta \neg \beta \phi_{1} \vee \phi_{2}\right)\right) \in F$. Then, the proof is identical to Case 1, when applied for $\left(\beta \neg \beta \phi_{1} \vee \phi_{2}\right)$.

Proof of Theorem 1. Recall that we define $\Phi_{n}$ as the closure of the set $\{\phi, \beta \phi \mid \phi \in$ $\left.\Phi_{n-1}\right\}$ with respect to $\neg, \vee$ and $\wedge$. It is straightforward verifying that $\Phi_{\infty}:=\bigcup_{n \geq 0} \Phi_{n}$ is such that $\Phi_{\infty}=\Phi$.

Thus, we prove the theorem by induction: We show that if the agent has faith in the truth axiom and introspection for all $\phi \in \Phi_{n}$, then he also has faith in the truth axiom and introspection for all $\phi^{\prime} \in \Phi_{n+1}$. This follows directly from Lemmas 2, 3 and 5 .

Proof of Theorem 2. Let $A_{3}-A_{5}$ be satisfied for all primitives at some $\omega \in \Omega_{r}$. By hypothesis, every proposition in $A$ is satisfied at $\omega$. Furthermore, the inference rules also hold locally at $\omega$ :

- $\left(\phi_{1} \wedge \phi_{2}\right) \in \omega$, if and only if $\phi_{1} \in \omega$ and $\phi_{2} \in \omega$

- If $\phi_{1} \in \omega$ and $\left(\phi_{1} \rightarrow \phi_{2}\right) \in \omega$, then $\phi_{2} \in \omega$

- $\beta \phi \in \omega$, for all $\phi \in F$ 
Thus, applying the steps of the proof of Theorem 1, locally at $\omega$, shows that the truth axiom and introspection is satisfied for all propositions at $\omega$. Therefore, all propositions in $F$ hold at $\omega$, which completes the proof.

\section{A.2 Proofs of Section 5}

Consider any sequence $\tilde{\beta}=\tau_{1}, \ldots, \tau_{n}, n \geq 1$, where for every $i=1, \ldots, n, \tau_{i}=\beta$ or $\tau_{i}=\neg \beta$. For such a sequence $\tilde{\beta}$, we define its parity $p(\tilde{\beta}) \in\{0,1\}$ as the parity of the number of occurrences of $\neg \beta$ in $\tilde{\beta}$. For instance, $p(\neg \beta \beta \neg \beta)=p(\beta)=0$, whereas $p(\beta \neg \beta)=p(\neg \beta \neg \beta \neg \beta)=1$, i.e., $p(\tilde{\beta})=0$ if the number of negations in $\tilde{\beta}$ is even, and $p(\tilde{\beta})=1$ otherwise.

Lemma 6. Let $\omega \in \Omega_{u}$. For any two sequences $\tilde{\beta}$ and $\tilde{\beta}^{\prime}$ such that $p(\tilde{\beta})=p\left(\tilde{\beta}^{\prime}\right)$ and for any proposition $\phi \in \Phi$, we have $\left(\tilde{\beta} \phi \leftrightarrow \tilde{\beta}^{\prime} \phi\right) \in F$.

Proof. It follows inductively from Theorem 1.

Corollary 3. Let $\omega \in \Omega_{u}$. For any two sequences $\tilde{\beta}$ and $\tilde{\beta}^{\prime}$ such that $p(\tilde{\beta})=p\left(\tilde{\beta}^{\prime}\right)$ and for any proposition $\phi \in \Phi$, we have $\beta(\tilde{\beta} \phi) \in \omega$, if and only if $\beta\left(\tilde{\beta}^{\prime} \phi\right) \in \omega$.

Proof. It follows directly from Lemma 6 and $A_{I}$.

Proof of Proposition 1. Let $(\neg \beta \phi \wedge \neg \beta \neg \beta \phi) \in \omega$, and suppose there is some $\phi^{\prime} \in B(\phi)$ such that $\beta \phi^{\prime} \in \omega$. By definition, $\beta \phi^{\prime}$ can be rewritten as $\beta \tilde{\beta} \phi$. If $p(\tilde{\beta})=0$ then $\left(\beta \phi^{\prime} \leftrightarrow \beta \phi\right) \in \omega$ which contradicts $\neg \beta \phi \in \omega$, whereas if $p(\tilde{\beta})=1$ then $\left(\beta \phi^{\prime} \leftrightarrow \beta \neg \beta \phi\right) \in \omega$ which contradicts $\neg \beta \neg \beta \phi \in \omega$. Hence, $u \phi \in \omega$ implies $\neg \beta \phi^{\prime} \in \omega$ for all $\phi^{\prime} \in B(\phi)$. The converse is straightforward.

Proof of Proposition 2. Suppose the contrary: The agent is aware of all primitive propositions. Then, the proof is identical to the one of Theorem 2. Note that in order to prove that introspection is well founded in $\Omega_{u}$, we do not require the truth axiom for the primitive to be well founded.

Proof of Proposition 3. By definition, if $\phi$ in $F$, then $\beta \phi \in \omega$ for every $\omega \in \Omega_{u}$. Assume that $\beta \phi \in \omega$ for every $\omega \in \Omega_{u}$, then $\beta \phi \in \omega$ for every $\omega \in \Omega_{5}$. Since the Truth axiom holds on $\Omega_{5}$, this implies that $\phi \in \omega$ for every $\omega \in \Omega_{5}$. Therefore $\phi$ is a tautology of $\Omega_{5}$, hence a theorem of S5, hence an element of $F$. 
Proof of Theorem 3. First we show that for every $\phi \in \Phi$ there is another proposition $\phi_{1} \in \Phi_{1}$ such that $\left(\phi \leftrightarrow \phi_{1}\right) \in F$, and therefore if $\omega$ and $\omega^{\prime}$ coincide in the truth value of $\phi_{1}$ they will also coincide in the truth value of $\phi$ - because of $A_{I}$ - which would suffice for the proof.

It follows from Lemmas 3,4 and 6 that for every $\phi_{n} \in \Phi_{n}$ there is some $\phi_{n-1} \in \Phi_{n-1}$ such that $\left(\phi_{n} \leftrightarrow \phi_{n-1}\right) \in F$. Continue inductively to obtain $\left(\phi_{n} \leftrightarrow \phi_{1}\right) \in F$, which completes the proof.

Proof of Theorem 4. Let $\phi \in \Phi_{1}$. For any $\omega \in \Omega_{u}$, there are two possibilities: either $u \phi \in \omega$, implying $\neg \beta \phi \in \omega$, or $a \phi \in \omega$, implying $(\beta \phi \vee \beta \neg \beta \phi) \in \omega$, and therefore $(\beta \phi \vee \neg \beta \phi) \in \omega$. The truth value of $\omega\left(\beta \phi_{0}\right)$ for all $\phi^{\prime} \in \Phi_{0}$ determines whether $\beta \phi \in \omega$ or $\neg \beta \phi \in \omega$. Thus, the truth value of $\omega\left(\beta \phi_{1}\right)$ is determined for all $\phi_{1} \in \Phi_{1}$, and therefore the proof follows directly from Theorem 3 .

\section{A.3 Proofs of Section 6}

Proof of Theorem 5. First, we show that $E_{\beta \phi} \subseteq B_{u} E_{\phi}$. Let $\omega \in E_{\beta \phi}$, which - by definition - is equivalent to $\beta \phi \in \omega$. Then, it follows from the definition of the possibility correspondence that $\phi \in \omega^{\prime}$ for all $\omega^{\prime} \in P(\omega)$, implying $\omega \in B E_{\phi}$. Furthermore, $\beta \phi \in \omega$ yields $\omega \in A E_{\phi}$, as required.

Now, we show that $E_{\beta \phi} \supseteq B_{u} E_{\phi}$. Let $\omega \in B_{u} E_{\phi}$. It follows from $\omega \in \Omega_{u}$ that the agent believes all propositions in $F$ at $\omega$, and therefore - by the definition of $P$ - all states $\omega^{\prime} \in P(\omega)$ are well founded. Hence, $P(\omega) \subseteq \Omega_{5}$. Furthermore, $A_{4}$ is satisfied at $\omega$. Hence, $P\left(\omega^{\prime}\right) \subseteq P(\omega)$, for all $\omega^{\prime} \in P(\omega)$ (Samet, 1990).

Now, suppose that $\neg \beta \phi \in \omega$. It follows from $\omega \in B_{u} E_{\phi}$ that the agent is aware of $\phi$ at $\omega$, implying $\beta \neg \beta \phi \in \omega$ (see Proposition 1). Hence, we obtain $\neg \beta \phi \in \omega^{\prime}$ for all $\omega^{\prime} \in P(\omega)$. It follows from Aumann (1999) that - since $\omega^{\prime} \in \Omega_{5}$ - there is some $\omega^{\prime \prime} \in P\left(\omega^{\prime}\right)$, such that $\neg \phi \in \omega^{\prime \prime}$. Finally, from $P\left(\omega^{\prime}\right) \subseteq P(\omega)$, it follows that $\omega^{\prime \prime} \in P(\omega)$, thus contradicting $\omega \in B E_{\phi}$, and therefore it also contradicts $\omega \in B_{u} E_{\phi}$, which completes the proof.

Proof of Proposition 5. For arbitrary $\phi_{1}, \phi_{2} \in \Phi$, let $E_{\phi_{1}}=E_{\phi_{2}}$, implying that $\left(\phi_{1} \leftrightarrow\right.$ $\left.\phi_{2}\right)$ is a tautology in $\Omega_{u}$, and therefore also in $\Omega_{5}$. Hence, $\left(\phi_{1} \leftrightarrow \phi_{2}\right)$ is a theorem in S5, implying that $\left(\phi_{1} \leftrightarrow \phi_{2}\right) \in F$. Finally, it follows from $R_{F}$ that $\beta\left(\phi_{1} \leftrightarrow \phi_{2}\right) \in \omega$, and therefore $\left(\beta \phi_{1} \leftrightarrow \beta \phi_{2}\right) \in \omega$ for all $\omega \in \Omega$, which completes the proof. 
Proof of Corollary 1. It follows by definition that $\Omega_{u}=E_{\phi \vee \neg \phi}$. Hence,

$$
\begin{array}{ccl}
B_{u} \Omega_{u} & = & B_{u} E_{\phi \vee \neg \phi} \\
& \stackrel{(\text { by Theorem 5) }}{=} & E_{\beta(\phi \vee \neg \phi)} \\
& \stackrel{\text { by } \left.B_{F}\right)}{=} & \Omega_{u}
\end{array}
$$

which completes the proof.

Proof of Corollary 2. It follows directly from the fact that every $\Omega_{5}$-tautology belongs to $F$, and therefore the agent believes it at all states in $\Omega_{u}$.

\section{References}

Aumann, R.J. (1976). Agreeing to disagree. Annals of Statistics 4, 1236-1239.

Aumann, R.J. (1997). Rationality and bounded rationality, Games and Economic Behavior 21, 2-14.

Aumann, R.J. (1999). Interactive epistemology I: knowledge, International Journal of Game Theory 28, 263-300.

Bacharach, M. (1999). Some extensions of a claim of Aumann in an axiomatic model of knowledge. Journal of Economic Theory 37, 167-190.

Battigalli, P., Bonanno, G. (1999). Recent results on belief, knowledge and the epistemic foundations of game theory, Research in Economics 53, 149-225.

Chellas, B.F. (1980). Modal logic: an introduction, Cambridge University Press, Cambridge.

Dekel, E., Lipman, B., Rustichini, A. (1998). Standard state-space models preclude unawareness, Econometrica 66, 159-173.

Fagin, R., Halpern, J.Y. (1988). Belief, awareness, and limited reasoning, Artificial Intelligence 34, 39-76.

Fagin, R., Halpern, J.Y., Moses, Y., Vardi, M.Y. (1995). Reasoning about knowledge, MIT press. 
FeinberG, Y. (2004). Subjective reasoning - Games with unawareness, Stanford Research Paper Series 1875.

Geanakoplos, J. (1989). Game theory without partitions, and applications to speculation and consensus, Cowles Foundation Discussion Paper 914, Yale University.

HALPERN, J.Y. (1995). The effect of bounding the number of primitive propositions and the depth of nesting on the complexity of modal logic, Artificial Intelligence 75, $361-372$.

Halpern, J.Y. (2001). Alternative semantics for unawareness, Games and Economic Behavior 37, 321-339.

Halpern, J.Y., RÊGO, L.C. (2008). Interactive unawareness revisited, Games and Economic Behavior 62, 232-262.

HARsanyi, J. (1967-68). Games with incomplete information played by Bayesian players, I-III. Management Science 14, 159-182, 320-334, 486-502.

Heifetz, A., Meier, M., Schipper, B. (2006). Interactive unawareness, Journal of Economic Theory 130, 78-94.

Heifetz, A., Meier, M., Schipper, B. (2008). A canonical model for interactive unawareness, Games and Economic Behavior 62, 304-324.

Heifetz, A., Meier, M., Schipper, B. (2009). Dynamic unawareness and rationalizable behavior. Discussion paper, UC Davis.

HintikkA, J. (1962). Knowledge and belief, Cornell University Press, Ithaca, NY.

KRIPKE, S. (1963). Semantic analysis of modal logic, Zeitschrift für Mathematische Logik und Grundlagen der Mathematik 9, 67-96.

LI, J. (2009). Information structures with unawareness, Journal of Economic Theory 144, 977-993.

LiPMAN, B. (1995). Information processing and bounded rationality: A survey, Canadian Journal of Economics 28, 42-67.

ModicA, S., Rustichini, A. (1994). Awareness and partitional information structures, Theory and Decision 37, 107-124. 
ModicA, S., Rustichini, A. (1999). Unawareness and partitional information structures, Games and Economic Behavior 27, 265-298.

Rubinstein, A. (1998). Modeling bounded rationality, MIT press, Cambridge, USA.

SAMET, D. (1990). Ignoring ignorance and agreeing to disagree, Journal of Economic Theory 52, 190-207.

Simon, H. (1955). A behavioral model of rational choice, The Quarterly Journal of Economics 69, 99-118. 